how to examine each part and how to diagnose soft tissue orthopædic lesions. His account of pathology is often controversial and none the worse for that. His methods of examination are meticulous and detailed, and no reader, however skilled, can fail to benefit from their study. The appendix on statistical analysis is much too short and no account is given of methods of case selection nor the criteria by which the results are assessed; this is a pity for unless the author devotes the same clinical thoroughness to evaluating results which he applies to clinical methods he cannot hope to convince the sceptics.

Many orthopædic surgeons will disagree with many of the statements in this book, but all of them will enjoy reading it and all will learn a lot from it. To every doctor interested in physical medicine (and in this age of discs this must include all general practitioners), and to every physiotherapist who treats orthopædic disorders the book is essential.

\section{Neurological Examination in Clinical Practice}

Edwin R. Bickerstaff. Pp. $x+357$, illustrated. Oxford: Blackwell Scientific Publications. I963. 50 .

This excellent book sets out in clear and precise detail the method of examination of the nervous system. This might be thought to have resulted in a somewhat dull recitation of instructions. This is far from being the case. The author has drawn on a wide clinical experience in order to indicate throughout the purpose of each test and how it can lead to a final diagnosis. Not only are details of the clinical examination given but also the proper use of such facilities as neuroradiology, electroencephalography, electromyography and otological tests is indicated. The book is so thorough in most fields that it is a pity that the details of cystometry are insufficient for an inexperienced person to be able to undertake it. Methods and rates of filling are omitted and a diagram of the apparatus would have been helpful.

The illustrations are of high standard, the production excellent and the book can be strongly recommended.

\section{Textbook of the Fundus of the Eye}

A. I. Ballantyne, Ll.D., M.D., F.R.F.P.S., and I. C. Michaelson, F.R.F.P.S., D.O.M.S., PH.D. Pp. xix + 520, illustrated. Edinburgh and London: E. \& S. Livingstone. 1962. £7.

This textbook is a welcome and valuable addition to the present selection of textbooks on the fundus oculi. The present form is largely attributable to Professor Michaelson, who since the death of Professor Ballantyne in 1953 has carried on with the work single-handed. The volume is made up of just over 500 pages, which includes 600 excellent illustrations, and these will partly explain the high price of the book. This factor will also serve to limit this largely for use as a reference book. The text is clear and simply written, the subject matter is interesting and comprehensive and every chapter is followed by a considerable reference section.

The opening chapters describe the means available for the investigation of the fundus by ophthalmoscopy, ophthalmodynanometry and electroretinography. The following chapters survey the vast realm of retinal vascular pathology and the feature of these chapters is the correlation of the ocular manifestations in the light of the disease as a whole, which is well demonstrated in the chapter on diabetes, where the close relationship of the retinopathy and the Kimmelstiel-Wilson syndrome is considered. Furthermore, the text is enhanced by excellent photomicrographs of the pathological changes. The insertion of a section on the treatment of hypertension which is outmoded and in parts inaccurate seems unnecessary in this book. Fundal manifestation of systemic diseases are considered in relation to the blood dyscrasias, the collagen diseases, the phakomatoses and the lipoidoses. These chapters would be of particular interest to the physician for both the material presented and the bibliography, but it is also valuable to know that here is a book where such topics as the association of colloid bodies in polyserositis or angioid streaks in Paget's disease can be found. The final chapters include a useful section on the clinical value of the electroretinogram, and the vitreous, choroid, and optic nerve are considered and the high standard of the rest of the book is maintained.

In conclusion, it may be said that this is a valuable and important book not only for the ophthalmologist, but for the physician as well, and is strongly recommended as being one of the best books on this subject.

\section{Diseases of the Nervous System}

Sir Francis Walshe. Tenth edition. Pp. xii + 381, illustrated. Edinburgh and London: E. \& S. Livingstone. 1963. 35 s.

This well-known textbook needs no introduction and little commendation; the appearance of the tenth edition is testimony enough to its value and popularity. The sub-title declares that the diseases are 'described for practitioners and students' and this surely is the secret of the book's success, for few can rival it in descriptions which make diseases spring to life and create mental pictures which never become blurred.

Although a valiant effort has been made to give therapeutics a place more in keeping with its present status in neurology, it cannot be said to have been wholly successful. Too much space is devoted to warnings against the dangers of over-treatment (which is hardly a besetting sin of neurologists) and too little and too imprecise information is given about therapy which can and should be employed. Nevertheless, the book remains one which no one interested in neurology can be without.

Therapist-Patient Expectancies in Psychotherapy

Arnold P. Goldstein. Pp. xvi + i4r. Oxford, London, New York and Paris: Pergamon Press. 1962. 30s.

It is said that doctors are at last becoming aware once more of the possible major significance of the doctor-patient relationship as an intervening variable affecting their management and care of patients. To those interested in this subject this book presents a study of the relationship from one particular viewpoint. The author attempts to evaluate the importance to therapeutic outcome of the treatment expectancies of the therapist and patient in psychotherapy. The subject matter is presented directly and clearly, and wide and useful reference is made to the relevant literature. The author's views appear particularly appropriate in his criticism of the concept of spontaneous remission. This particular chapter serves to highlight the possible importance of the doctor-patient relationship. The chapter on the placebo effect, which also contains some useful references to the phenomena of transference cure, effectively embodies the author's approach.

This whole research field is a particularly exciting one, since it involves the study of human relationships. Research at the level of patient expectancy will probably be complicated by the fact that the human quality of 